\title{
Costo real de producción del litro de leche, en pequeños ganaderos de la comunidad de Sivicusig, cantón Sigchos, Ecuador
}

\author{
Adolfo Cevallos Polanco \\ clc.asesoriayproyectos@gmail.com \\ Asesor de proyectos. MSc. Profesional Independiente. \\ Cotopaxi- Ecuador. \\ María Verónica Taipe Taipe \\ veritott@hotmail.com \\ Investigador. MSc. Profesional Independiente. \\ Santo Domingo - Ecuador. \\ Francisco Iván Caiza de la Cueva \\ fcaiza@biogensa.com.ec \\ Director científico. $\mathrm{PhD}$. Centro de investigación y biotecnologías \\ del Ecuador "CIBRAE", ProduBiogensa, Machachi - Ecuador.
}

\section{RESUMEN}

El pequeño productor, sin disponer de los elementos necesarios para producir, no considera su sistema de producción como una actividad económica, por tal motivo no aplica la contabilidad cuya finalidad es registrar sistemáticamente todos los rubros de egresos e ingresos que se generen diariamente y que al final de un período, considere su ganancia o pérdida y realice los ajustes financieros necesarios cuando así lo requiera. El Objetivo de la investigación fue determinar el costo de producción real del litro de leche, para lo cual se extrajo información de 17 ganaderos dedicados a la producción de leche, del análisis socioeconómico de la comunidad de Sivicusig, cantón Sigchos, que constituyeron el insumo para establecer el precio de venta, los costos de producción y el análisis de rentabilidad. La estimación del costo de producción del litro de leche fue realizada mediante el concepto de costos diferenciales (costos fijos y costos variables). En la comunidad hay una producción promedio de 6352,9 litros de leche con una desviación estándar de 3539,9 litros, el costo de producción promedio por litro de acuerdo a los productores de 0,21 dólares con una desviación de 0,10 dólares, sin embargo, el costo de producción real si llevaran contabilidad es de 0,43 dólares con una desviación de 0,19 dólares. El margen de utilidad bruta (MUB) demuestra que los productores pierden en sus actividades, pues tienen un déficit de 16,8 dólares. El margen porcentual 
(MP) indica que alcanzan al 2,8\% en promedio, de pérdidas en la producción. Los pequeños productores al no ver la actividad pecuaria, como una empresa, no llevan los registros contables y por ello desconocen los costos reales de producción, razón por la cual, no identifican si su actividad es rentable, no crecen y no llegan a ser competitivos.

Palabras clave: producción; leche; costos; registros contables; rentabilidad; sivicusig. 


\title{
Real cost of production per liter of milk, in small farmers in the community of Sivicusig, Sigchos canton, Ecuador
}

\begin{abstract}
The small producer, without having the necessary elements to produce, does not consider his production system as an economic activity, for this reason he does not apply accounting whose purpose is to systematically record all the items of expenses and income that are generated daily and that at the end of a period, consider your profit or loss and make the necessary financial adjustments when required. The objective of the research was to determine the real production cost of the liter of milk, for which information was extracted from 17 farmers dedicated to milk production, from the socioeconomic analysis of the community of Sivicusig, canton Sigchos, which constituted the input for establish the sale price, production costs and profitability analysis. The estimation of the production cost of a liter of milk was carried out through the concept of differential costs (fixed costs and variable costs). In the community there is an average production of 6352.9 liters of milk with a standard deviation of 3539.9 liters, the average production cost per liter according to the producers of 0.21 dollars with a deviation of 0.10 dollars, however, the actual production cost if they kept accounting is $\$ 0.43$ with a deviation of $\$ 0.19$. The gross profit margin (MUB) shows that producers lose in their activities, since they have a deficit of 16.8 dollars. The percentage margin (MP) indicates that they reach $2.8 \%$ on average, of losses in production. Because small producers do not see the livestock activity, such as a company, they do not keep accounting records and therefore do not know the real costs of production, which is why they do not identify if their activity is profitable, they do not grow and do not become competitive.
\end{abstract}

Keywords: production; Milk; Costs; Accounting records; Cost effectiveness; Sivicusig

Artículo recibido: 10. Junio. 2021 Aceptado para publicación: 16. Julio. 2021 Correspondencia: veritott@hotmail.com Conflictos de Interés: Ninguna que declarar 


\section{INTRODUCCIÓN}

En la actualidad, momento en que el mundo entero es azotado por el COVID 19 y atraviesa una debacle económica a nivel mundial, se vuelve más evidente que nunca, la necesidad de la subsistencia mediante el fortalecimiento no solo de los sistemas sanitarios, sino a la par de la producción agropecuaria que posibilite el fortalecimiento de la población. Esto se alcanza con una adecuada y diversificada producción de frutas, hortalizas, legumbres, granos, etc. y de un producto indispensable en la alimentación humana como es la leche y todos sus derivados.

Entonces la producción agropecuaria es prioridad actual de los gobiernos aportando con todos los factores requeridos en forma planificada y metódica, que permitan en el caso de la producción láctea, hacer más eficientes a los productores, elevar los estándares de calidad, los volúmenes de producción, sistemas de comercialización, entre otros. Para lograr este propósito son necesarios capacitación, asistencia técnica permanente, apoyo a la organización, entrega de crédito con adecuados intereses y que al final el pequeño y mediano productor y su familia obtenga réditos acordes a su esfuerzo.

Sin embargo, los costos de producción afectan negativamente la rentabilidad de la operación, estos varían en función del nivel tecnológico aplicado en la granja, la disponibilidad de mano de obra, agua, forraje para el ganado, la genética animal, entre otros. Nueva Zelanda registra los costos de producción más bajos en el ámbito mundial. Al contrario, Japón, Noruega y Suiza presentan altos costos de producción, debido a la falta de tierras para el ganado (Gómez, et al., 2017). Los costos de producción en Ecuador varían según la región y el sistema de producción, así por ejemplo en la costa alcanza \$0,70 (El Universo, 2019) en promedio es de \$ 0,38 el litro de leche. Los rubros de alimentación y mano de obra son los más importantes y representan del 75 al 80\%.

Al momento el pequeño productor, sin disponer de los elementos mencionados, no considera su sistema de producción como una actividad económica tal como lo cataloga una pequeña, mediana o gran empresa y por tal motivo no aplica la CONTABILIDAD cuyo objetivo es registrar sistemáticamente todos los rubros de egresos e ingresos que se generen diariamente y que al final de un período, sea semanal, mensual, semestral o anual, considere su ganancia o pérdida en el negocio y realice los ajustes financieros necesarios cuando así lo requiera. 
Esta situación no permite determinar con mayor exactitud, en control, corrección y evaluación de los costos, ingresos, egresos e inversiones, a fin de cumplir con las disposiciones legales existentes para el pago de impuestos y para la planificación integral del sector agropecuario en general. Con estos antecedentes es necesario aclarar que la única forma de conocer y saber con exactitud el costo de producción del litro de leche en su finca, es contando con una contabilidad real que refleje la verdadera situación de la empresa. Y capacitar al productor lechero para que, antes de nada, sepa exactamente cuánto es el costo de producción real.

Es por ello, que, en esta investigación, se pretendió determinar el costo real de producción de leche, para que el productor entienda su situación económica y financiera en los sistemas de producción de leche. Asimismo, la investigación espera aportar para el conocimiento de un manejo eficiente de los registros contables, por parte del empresario sin importar el tamaño de emprendimiento sino más bien viabilizando su efectividad.

\section{ESTRATEGIAS METODOLÓGICAS O MATERIALES Y MÉTODOS}

\subsection{Ubicación}

Sivicusig, es una zona de la serranía ecuatoriana, que pertenece al cantón Sigchos, provincia de Cotopaxi, con altitudes que varían entre 2800 - 3100 m.s.n.m., 78 53'06.2” de longitud Oeste y $00^{\circ} 49^{\prime}$ 59.1" latitud Sur (Google Maps, 2021). Limita: al Norte: Cantón Santo Domingo; al Sur: Cantón Pujilí; al Este: Cantón Mejía y Cantón Latacunga y al Oeste: Cantón La Maná (GADMSigchos, 2015).

\subsection{Características Climáticas}

La comunidad de Sivicusig, cuenta con tres pisos climáticos que son: Tropical Megatérmico Húmedo, Ecuatorial Mesotérmico Semi-Húmedo y Ecuatorial de Alta Montaña, La distribución de las lluvias en el cantón varía mucho de una zona a otra, la precipitación media anual en las partes media y alta está entre los 500 a $1500 \mathrm{~mm}$, en tanto que para las zonas del subtrópico se presentan valores entre los 2000 a $3000 \mathrm{~mm}$. La temperatura varía entre 0 y $22{ }^{\circ} \mathrm{C}$ (GADMSigchos, 2015).

\subsection{Procedimiento}

La información fue extraída del análisis socioeconómico de la comunidad de Sivicusig, cantón Sigchos, que constituyeron el insumo para establecer el precio de venta y los costos de producción, asociado con la producción de leche que se presenta en este trabajo.

\subsection{1 Áreas dedicadas a la ganadería}


Sivicusig, dispone de 51,13 ha dedicadas a la ganadería de las cuales, 11,18 ha son de mezcla forrajera (Ray grass, trébol, pasto azul, alfalfa, vicia y avena forrajera), 33,65 ha de pasto natural (Kikuyo) y 3,3 ha de Holco (Cuadro N.- 1), además de los servicios básicos requeridos en las zonas rurales, carreteras de segundo y tercer orden.

Tabla $\mathbf{N}^{\mathbf{0}}$ 1: Héctareas (ha) dedicadas a la ganadería, de la comunidad Sivicusig, cantón Sigchos.

\begin{tabular}{ccccc}
\hline Propietario & Mezcla Forrajera & Kikuyo & Holco & Total \\
\hline 1 & 0 & 6.85 & 0 & 6.85 \\
2 & 1.7 & 0 & 0 & 1.7 \\
3 & 0 & 5.4 & 0 & 5.4 \\
4 & 0.5 & 3 & 0 & 3.5 \\
5 & 0.7 & 9.3 & 0 & 10 \\
6 & & 0 & 3.3 & 3.3 \\
7 & 0 & 3.3 & 0 & 3.3 \\
8 & 1.5 & 0 & 0 & 1.5 \\
9 & 0 & 5 & 0 & 5 \\
10 & 2.47 & 0 & 0 & 2.47 \\
11 & 0.3 & 0 & 0 & 0.3 \\
12 & 1.8 & 0 & 0 & 1.8 \\
13 & 0 & 0.56 & 0 & 0.56 \\
14 & 0.71 & 0 & 0 & 0.71 \\
15 & 1.5 & 0.24 & 0 & 1.74 \\
16 & 0 & 0.75 & 0 & 0.75 \\
17 & 0 & 2.25 & 0 & 2.25 \\
\hline Total & 11.18 & 36.65 & 3.3 & $\mathbf{5 1 . 1 3}$ \\
\hline
\end{tabular}

Fuente: Elaborado por Adolfo Cevallos Polanco

\subsubsection{Población}

En la comunidad de Sivicusig, cantón Sigchos, se encuentran establecidos 17 pequeños ganaderos, dedicados a la producción de leche, a quienes se ha aplicado la encuesta para obtener información y determinar el costo de producción real del litro de leche.

\subsubsection{Animales}

En la comunidad se registró la existencia de 5 toros reproductores, 12 novillos, 43 vacas en producción, 26 vacas secas, 4 vaconas vientre, 3 vaconas fierro, 8 vaconas media, y 29 terneras de la raza Holstein Friessen cruzado (Cuadro N.- 2), adaptadas a la zona.

Tabla $\mathbf{N}^{\mathbf{0}}$ 2: Composición de los hatos ganaderos, de la comunidad Sivicusig, cantón Sigchos. 


\begin{tabular}{|c|c|c|c|c|c|c|c|c|c|c|c|c|c|c|c|c|c|c|}
\hline \multirow{2}{*}{ Categoría } & \multicolumn{17}{|c|}{ Propietario } & \multirow[t]{2}{*}{ Total } \\
\hline & 1 & 2 & 3 & 4 & 5 & 6 & 7 & 8 & 9 & 10 & 11 & 12 & 13 & 14 & 15 & 16 & 17 & \\
\hline Toros & 1 & 1 & & & & & 1 & 1 & 1 & & & & & & & & & 5 \\
\hline Novillos & 4 & & 5 & & & 1 & & & & 2 & & & & & & & & 12 \\
\hline $\begin{array}{l}\text { Vacas en } \\
\text { producción }\end{array}$ & 4 & 4 & 5 & 1 & 5 & 2 & 2 & 2 & 3 & 2 & 1 & 3 & 2 & 1 & 4 & 1 & 1 & 43 \\
\hline Vacas secas & 3 & 1 & & & 1 & 3 & 2 & 2 & 1 & 2 & 2 & & & 1 & 4 & & 4 & 26 \\
\hline Vaconas vientre & 4 & & & & & & & & & & & & & & & & & 4 \\
\hline Vaconas fierro & & & 2 & & & & & & & & & & & & 1 & & & 3 \\
\hline Vaconas media & & 2 & & & 1 & & 3 & 2 & & & & & & & & & & 8 \\
\hline Terneras & 4 & & 5 & & 5 & 2 & 2 & & 3 & & 2 & & 2 & & 3 & 1 & & 29 \\
\hline Total Animales & 20 & 8 & 17 & 1 & 12 & 8 & 10 & 7 & 8 & 6 & 5 & 3 & 4 & 2 & 12 & 2 & 5 & 130 \\
\hline
\end{tabular}

Fuente: Elaborado por Adolfo Cevallos Polanco

\subsubsection{Alimentación}

La alimentación se basa en los pastizales (mezcla forrajera y pasto natural) que se encuentran en la zona, a las vacas en producción, le proporcionan una suplementación a base de balanceado en el momento del ordeño, el suministro agua es ad libitum.

\subsubsection{Producción de leche}

Se registró, el promedio de producción de leche obtenida, tanto del ordeño de la mañana, como de la tarde, de acuerdo a la información proporcionada por los ganaderos, y se procedió a multiplicar por el periodo de lactancia promedio de 270 días.

\subsubsection{Ingresos por venta de leche}

El Sr. Eddy Pesantes, subsecretario de Producción Pecuaria del Ministerio de Agricultura y Ganadería (MAG), señaló que los productores recibirán \$ 0,42 por la venta del litro de leche, que es el precio oficial conforme indica el Acuerdo 394 vigente desde 2013 (El telégrafo, 2018; Ekos, 2019; El Mercurio, 2020). El precio de venta del producto se expresó en unidades monetarias (\$) y fue considerado el que, en ese momento estaba en el mercado.

\subsubsection{Costo de producción de leche}


La estimación del costo de producción del litro de leche fue realizada mediante el concepto de costos diferenciales (costos fijos y costos variables). Los costos fijos incluyeron aquellos costos independientes del área de operación, tales como mano de obra, arrendamiento del terreno, servicios públicos, visita veterinaria, depreciación de instalaciones, equipos y animales. La depreciación de instalaciones y equipos se estimó considerando la vida útil: 15 años para el establo, la sala y los equipos de ordeño; 10 para las canecas y 5 para las fumigadoras. La depreciación de los animales se calculó como [(valor comercial en la zona - valor residual) / vida productiva de la vaca (7 años)]. El valor residual corresponde al valor de venta del animal de descarte. Todos los criterios de depreciación cumplieron con los parámetros establecidos en las normas internacionales de información financiera (NIIF, 2012). Los costos variables incluyeron aquellos costos que se modificaron de acuerdo con el volumen de producción, esto es, insumos para el mantenimiento de los pastos, la sanidad y la alimentación animal. Los costos de alimentación incluyeron el costo del forraje y de los suplementos. El costo del forraje (\$/kg MS) se calculó a partir de la cantidad de insumos agropecuarios utilizados por pastoreo (fertilizante, pesticida) y de la mano de obra requerida para su aplicación (Osorio, 2004). Cabe resaltar que los ganaderos de la comunidad mantienen un sistema de explotación tradicional, con limitada tecnología y no llevan un registro contable, por lo que un gran porcentaje, consideran en los costos fijos ciertos servicios, así como el impuesto predial y en los costos variables el mantenimiento del pasto y de los animales.

\subsubsection{Análisis de rentabilidad}

La rentabilidad se valoró a partir de cuatro parámetros, que son: margen de utilidad bruta (MUB), margen porcentual (MP), (Wolf, 2010 y Britt, et al., 2003).

El MUB se obtuvo por diferencia entre los ingresos por venta y el costo de producción de leche.

El MP resultó de la relación (MUB/ingresos por venta) $\times 100$. 


\section{RESULTADOS Y DISCUSIÓN}

\subsection{Resultados}

\subsubsection{Producción de leche}

El promedio de producción diaria por vaca de los ganaderos de la comunidad Sivicusig es de 9,4 litros, con una desviación estándar de 0,5. Sumando la producción en un periodo de lactancia de todos los ganaderos se obtuvo un total de 108000 litros de leche.

Tabla $N^{\mathbf{0}}$ 3: Producción de leche en la comunidad Sivicusig, cantón Sigchos.

\begin{tabular}{ccccc}
\hline Propietario & $\begin{array}{c}\text { Vacas En } \\
\text { Producción }\end{array}$ & $\begin{array}{c}\text { Producción } \\
\text { Vaca Día }\end{array}$ & $\begin{array}{c}\text { Producción Vaca } \\
\text { Lactancia }\end{array}$ & Producción Total \\
\hline 1 & 4 & 10 & 2700 & 10800 \\
2 & 4 & 9 & 2430 & 9720 \\
3 & 5 & 9 & 2430 & 12150 \\
4 & 1 & 10 & 2700 & 2700 \\
5 & 5 & 9 & 2430 & 12150 \\
6 & 2 & 9 & 2430 & 4860 \\
7 & 2 & 9 & 2430 & 4860 \\
8 & 2 & 9 & 2430 & 4860 \\
9 & 3 & 9 & 2430 & 7290 \\
10 & 2 & 10 & 2700 & 5400 \\
11 & 1 & 10 & 2700 & 2700 \\
12 & 3 & 9 & 2430 & 7290 \\
13 & 2 & 9 & 2430 & 4860 \\
14 & 1 & 10 & 2700 & 2700 \\
15 & 4 & 10 & 2700 & 10800 \\
16 & 1 & 9 & 2430 & 2430 \\
17 & 1 & 9 & 2430 & 2430 \\
\hline Total & 43 & 159 & 42930 & 108000 \\
\hline Promedio & 2,5 & 9,4 & 2525,3 & 6352,9 \\
\hline Desviación & 1,4 & 0,5 & 133,0 & 3539,9 \\
estándar & & & & \\
\hline & & 9 & 9 & \\
\hline
\end{tabular}

Fuente: Elaborado por Adolfo Cevallos Polanco

\subsubsection{Ingresos por venta de leche}

El ingreso por venta de leche se determinó del producto entre la producción de leche en un periodo de lactancia y el precio oficial del litro de leche, el resultado total es de 45360 dólares, el promedio de ingresos es de 2668,2 dólares con una desviación estándar de 1486,8 dólares. 
Tabla N 4: Ingresos por venta de leche en la comunidad Sivicusig, cantón Sigchos.

\begin{tabular}{cccc}
\hline Propietario & $\begin{array}{c}\text { Producción Por Periodo } \\
\text { De Lactancia }\end{array}$ & $\begin{array}{c}\text { Precio De Venta Por } \\
\text { Litro }\end{array}$ & \begin{tabular}{c} 
Ingresos Totales \\
\hline 1
\end{tabular} In800 $_{2}$ \\
9720 & 0,42 & 4536,0 \\
3 & 12150 & 0,42 & 4082,4 \\
4 & 2700 & 0,42 & 5103,0 \\
5 & 12150 & 0,42 & 1134,0 \\
6 & 4860 & 0,42 & 5103,0 \\
7 & 4860 & 0,42 & 2041,2 \\
8 & 4860 & 0,42 & 2041,2 \\
9 & 7290 & 0,42 & 2041,2 \\
10 & 5400 & 0,42 & 3061,8 \\
11 & 2700 & 0,42 & 2268,0 \\
12 & 7290 & 0,42 & 1134,0 \\
13 & 4860 & 0,42 & 3061,8 \\
14 & 2700 & 0,42 & 2041,2 \\
15 & 10800 & 0,42 & 1134,0 \\
16 & 2430 & 0,42 & 4536,0 \\
17 & 2430 & 0,42 & 1020,6 \\
\hline Total & 108000 & 0,42 & 1020,6 \\
\hline Promedio & 6352,9 & & 45360,0 \\
\hline Desviación & & & 2668,2 \\
estándar & 3539,9 & & 1486,8 \\
\hline Fuente: Elate & & \\
\hline
\end{tabular}

Fuente: Elaborado por Adolfo Cevallos Polanco

\subsubsection{Costo de producción}

El costo de producción promedio del litro de leche tal cual lo consideran los ganaderos de la comunidad de Sivicusig es de 0,21 dólares con una desviación estándar de 0,10 dólares, sin embargo, el costo promedio de producción real es de 0,43 dólares con una desviación estándar de 0,19 dólares.

Tabla $\mathbf{N}^{\circ}$ 5: Costo de producción del litro de leche que consideran los ganaderos en la comunidad Sivicusig, cantón Sigchos.

\begin{tabular}{|c|c|c|c|c|c|c|}
\hline \multirow[b]{2}{*}{ Propietario } & \multicolumn{2}{|c|}{ Costos Variables } & \multicolumn{2}{|c|}{ Costos Fijos } & \multirow[b]{2}{*}{ Costo total } & \multirow{2}{*}{$\begin{array}{c}\text { Costo de } \\
\text { producción } \\
\text { por litro }\end{array}$} \\
\hline & $\begin{array}{c}\text { Mantenimiento } \\
\text { de pastos }\end{array}$ & $\begin{array}{c}\text { Mantenimiento } \\
\text { de animales }\end{array}$ & Servicios & Impuestos & & \\
\hline 1 & 1885,7 & 923,1 & 184,6 & 67,0 & 3060,3 & 0,28 \\
\hline 2 & 468,0 & 369,2 & 73,8 & 16,6 & 927,7 & 0,10 \\
\hline 3 & 1486,5 & 784,6 & 156,9 & 52,8 & 2480,9 & 0,20 \\
\hline 4 & 963,5 & 46,2 & 9,2 & 34,2 & 1053,1 & 0,39 \\
\hline 5 & 2752,8 & 553,8 & 110,8 & 97,8 & 3515,2 & 0,29 \\
\hline
\end{tabular}




\begin{tabular}{ccccccc}
6 & 908,4 & 369,2 & 73,8 & 32,3 & 1383,8 & 0,28 \\
7 & 908,4 & 461,5 & 92,3 & 32,3 & 1494,5 & 0,31 \\
8 & 412,9 & 323,1 & 64,6 & 14,7 & 815,3 & 0,17 \\
9 & 1376,4 & 369,2 & 73,8 & 48,9 & 1868,4 & 0,26 \\
10 & 679,9 & 276,9 & 55,4 & 24,2 & 1036,4 & 0,19 \\
11 & 82,6 & 230,8 & 46,2 & 2,9 & 362,4 & 0,13 \\
12 & 495,5 & 138,5 & 27,7 & 17,6 & 679,3 & 0,09 \\
13 & 154,2 & 184,6 & 36,9 & 5,5 & 381,2 & 0,08 \\
14 & 195,4 & 92,3 & 18,5 & 6,9 & 313,2 & 0,12 \\
15 & 479,0 & 553,8 & 110,8 & 17,0 & 1160,6 & 0,11 \\
16 & 206,5 & 92,3 & 18,5 & 7,3 & 324,6 & 0,13 \\
17 & 619,4 & 230,8 & 46,2 & 22,0 & 918,3 & 0,38 \\
\hline Total & 14075,0 & 6000,0 & 1200,0 & 500,0 & 21775,0 & 3,51 \\
\hline Promedio & 827,9 & 352,9 & 70,6 & 29,4 & 1280,9 & 0,21 \\
\hline Desviación & & & & & \\
estándar & 706,4 & 243,1 & 48,6 & 25,1 & 950,8 & 0,10 \\
\hline
\end{tabular}

Fuente: Elaborado por Adolfo Cevallos Polanco 
Tabla N 6: Costo de producción real del litro de leche en la comunidad Sivicusig, cantón Sigchos.

\begin{tabular}{|c|c|c|c|c|c|c|c|c|c|c|}
\hline \multirow[b]{2}{*}{ Propietario } & \multicolumn{4}{|c|}{ Costos Variables } & \multicolumn{4}{|c|}{$\begin{array}{l}\text { Costos fijos } \\
\end{array}$} & \multirow[b]{2}{*}{$\begin{array}{c}\text { Costo } \\
\text { total }\end{array}$} & \multirow[b]{2}{*}{$\begin{array}{c}\text { Costo de } \\
\text { producción } \\
\text { por litro }\end{array}$} \\
\hline & $\begin{array}{c}\text { Establecimiento } \\
\text { de pastos }\end{array}$ & $\begin{array}{l}\text { Mantenimiento } \\
\text { de pastos }\end{array}$ & $\begin{array}{l}\text { Mantenimiento } \\
\text { de animales }\end{array}$ & $\begin{array}{l}\text { Asistencia } \\
\text { técnica }\end{array}$ & $\begin{array}{c}\text { Mantenimiento } \\
\text { de } \\
\text { construcciones }\end{array}$ & $\begin{array}{c}\text { Mantenimiento } \\
\text { de maquinaria } \\
\text { y equipo }\end{array}$ & Servicios & Impuestos & & \\
\hline 1 & 744,9 & 1885,7 & 923,1 & 223,3 & 1550,8 & 781,1 & 184,6 & 67,0 & 11760,3 & 0,59 \\
\hline 2 & 184,9 & 468,0 & 369,2 & 223,3 & 620,3 & 193,8 & 73,8 & 16,6 & 7549,9 & 0,22 \\
\hline 3 & 587,2 & 1486,5 & 784,6 & 279,1 & 1318,2 & 615,7 & 156,9 & 52,8 & 10681,0 & 0,43 \\
\hline 4 & 380,6 & 963,5 & 46,2 & 55,8 & 77,5 & 399,1 & 9,2 & 34,2 & 7366,1 & 0,73 \\
\hline 5 & 1087,4 & 2752,8 & 553,8 & 279,1 & 930,5 & 1140,2 & 110,8 & 97,8 & 12352,4 & 0,57 \\
\hline 6 & 358,8 & 908,4 & 369,2 & 111,6 & 620,3 & 376,3 & 73,8 & 32,3 & 8250,8 & 0,59 \\
\hline 7 & 358,8 & 908,4 & 461,5 & 111,6 & 775,4 & 376,3 & 92,3 & 32,3 & 8516,7 & 0,64 \\
\hline 8 & 163,1 & 412,9 & 323,1 & 111,6 & 542,8 & 171,0 & 64,6 & 14,7 & 7203,8 & 0,37 \\
\hline 9 & 543,7 & 1376,4 & 369,2 & 167,4 & 620,3 & 570,1 & 73,8 & 48,9 & 9169,9 & 0,52 \\
\hline 10 & 268,6 & 679,9 & 276,9 & 111,6 & 465,2 & 281,6 & 55,4 & 24,2 & 7563,5 & 0,40 \\
\hline 11 & 32,6 & 82,6 & 230,8 & 55,8 & 387,7 & 34,2 & 46,2 & 2,9 & 6272,8 & 0,32 \\
\hline 12 & 195,7 & 495,5 & 138,5 & 167,4 & 232,6 & 205,2 & 27,7 & 17,6 & 6880,3 & 0,20 \\
\hline 13 & 60,9 & 154,2 & 184,6 & 111,6 & 310,2 & 63,9 & 36,9 & 5,5 & 6327,7 & 0,19 \\
\hline 14 & 77,2 & 195,4 & 92,3 & 55,8 & 155,1 & 81,0 & 18,5 & 6,9 & 6082,2 & 0,25 \\
\hline 15 & 189,2 & 479,0 & 553,8 & 223,3 & 930,5 & 198,4 & 110,8 & 17,0 & 8101,9 & 0,25 \\
\hline 16 & 81,6 & 206,5 & 92,3 & 55,8 & 155,1 & 85,5 & 18,5 & 7,3 & 6102,5 & 0,29 \\
\hline 17 & 244,7 & 619,4 & 230,8 & 55,8 & 387,7 & 256,6 & 46,2 & 22,0 & 7263,0 & 0,77 \\
\hline Total & 5560,0 & 14075,0 & 6000,0 & 2400,0 & 10080,0 & 5830,0 & 1200,0 & 500,0 & 137445,0 & 7,34 \\
\hline Promedio & 327,1 & 827,9 & 352,9 & 141,2 & 592,9 & 342,9 & 70,6 & 29,4 & 8085,0 & 0,43 \\
\hline $\begin{array}{l}\text { Desviación } \\
\text { Estándar }\end{array}$ & 279,1 & 706,4 & 243,1 & 79,2 & 408,4 & 292,6 & 48,6 & 25,1 & 1906,3 & 0,19 \\
\hline
\end{tabular}

Fuente: Elaborado por Adolfo Cevallos Polanco 


\subsubsection{Análisis de rentabilidad}

Para el caso del análisis de rentabilidad considerado por el productor se observa un promedio de 1334,4 dólares con una desviación estándar de 954,4 dólares, lo que quisiera decir que los productores de leche en la comunidad tienen utilidad, sin embargo, en el análisis de rentabilidad real, los productores pierden en promedio 16,8 dólares, es decir que su negocio no es rentable,

Tabla No 7: Análisis de rentabilidad de la producción láctea en la comunidad Sivicusig, cantón Sigchos,

\begin{tabular}{ccccc}
\hline Productor & Considerado por el productor & \multicolumn{2}{c}{ Real } \\
\hline 1 & MUB $^{\mathbf{1}}$ & MP $^{\mathbf{2}}$ & MUB & MP \\
2 & 1492,3 & 32,9 & $-1824,3$ & $-40,2$ \\
3 & 3003,1 & 73,6 & 1932,5 & 47,3 \\
4 & 2635,3 & 51,6 & $-178,0$ & $-3,5$ \\
5 & 43,6 & 3,8 & $-832,1$ & $-73,4$ \\
6 & 1547,9 & 30,3 & $-1849,4$ & $-36,2$ \\
7 & 665,4 & 32,6 & $-809,6$ & $-39,7$ \\
8 & 554,7 & 27,2 & $-1075,5$ & $-52,7$ \\
9 & 1092,1 & 53,5 & 237,4 & 11,6 \\
10 & 1205,6 & 39,4 & $-708,1$ & $-23,1$ \\
11 & 1011,2 & 44,6 & 104,5 & 4,6 \\
12 & 744,8 & 65,7 & 261,2 & 23,0 \\
13 & 2222,0 & 72,6 & 1581,5 & 51,7 \\
14 & 1661,4 & 81,4 & 1113,5 & 54,6 \\
15 & 757,5 & 66,8 & 451,8 & 39,8 \\
16 & 3242,1 & 71,5 & 1834,1 & 40,4 \\
17 & 697,9 & 68,4 & 318,1 & 31,2 \\
\hline Total & 107,8 & 10,6 & $-842,4$ & $-82,5$ \\
\hline Promedio & 22684,6 & 826,4 & $-285,0$ & $-47,1$ \\
\hline Desviación & 1334,4 & 48,6 & $-16,8$ & $-2,8$ \\
estándar & 954,4 & 23,3 & 1166,5 & 45,2 \\
\hline
\end{tabular}

${ }^{1}$ MUB (margen de utilidad bruta)

${ }^{2} \mathrm{MP}$ (margen porcentual)

\subsection{Discusión}

Fuente: Elaborado por Adolfo Cevallos Polanco

La producción de leche es vital para las familias campesinas de la comunidad de Sivicusig, las cuales en su mayoría disponen de menos 2 ha, sin acceso al riego y sobreviven en la pobreza e inseguridad, estas unidades agropecuarias no son tecnificadas y no conocen que es un registro contable, Del diagnóstico realizado, el promedio de producción por vaca/día es de 9,4 litros, considerando la producción en un periodo de 
lactancia de 270 días alcanzaron a 6352,9 litros de leche promedio y una desviación estándar de 3539,9 litros, Salazar y Cochet (2016) mencionan que la producción varía de acuerdo a los sistemas de producción, estudios realizados en el Carchi, reflejan producciones promedio de: 11, 16, 14 y 9 litros/vaca/día, en haciendas extensivas, haciendas recientemente constituidas, campesinos exguasipungueros y campesinos herederos respectivamente, Torres (2018) presenta la producción promedio de: 21, 12 y 6 litros/vaca/día para el productor grande, mediano y pequeño respectivamente en sus estudios realizados en el cantón Cayambe,

El costo de producción, afecta directamente las utilidades del emprendedor y es una variable importante en la toma de decisiones para ser competitivo, En este sentido es necesario establecer el costo de producción sobre una medida comparable con cualquier otro productor, esto es, en cuanto al costo por litro de leche producido, la única forma de determinar con exactitud el costo de producción del litro de leche en la finca, es contando con una contabilidad real que refleje la verdadera situación de la empresa, El costo de producción considerado por los productores de la comunidad Sivicusig es de $\$ 0,21$ dólares con una desviación estándar de $\$ 0,10$, pues al no llevar un registro de los egresos ni tampoco una contabilidad, solo consideran aspectos básicos como mantenimiento de pasto y animales, servicios básicos e impuestos, Mientras el costo de producción real alcanza un promedio de $\$ 0,43$ con una desviación estándar de $\$ 0,19$ sin considerar, algunas variables dentro de los costos fijos y variables que se toman en cuenta para determinar si una empresa es rentable o no, Según Torres (2018), el costo de producción por litro de leche es de; 33, 35 y 76 centavos de dólar para para el productor grande, mediano y pequeño respectivamente,

La rentabilidad se refiere a la habilidad que tiene una empresa para generar un retorno económico neto con el uso de unos recursos o activos en particular (Hadley, Harsh y Wolf, 2002), En este estudio, el costo total excedió el ingreso total, disminuyendo la rentabilidad, El margen de utilidad bruta (MUB) demuestra que los productores pierden en sus actividades, pues tienen un déficit de 16,8 dólares, El margen porcentual (MP) indica que alcanzan al 2,8\% de pérdidas en la producción, Para Fedegan (2004), solo el $26 \%$ de los productores de leche de países industrializados como EEUU alcanzan a cubrir sus costos de operación, destacándose la importancia del apoyo del estado norteamericano 
al garantizar un nivel de vida para el productor, mediante la puesta en práctica de políticas de sustentación de precios a través de subsidios a la producción.

\section{CONCLUSIÓN O CONSIDERACIONES FINALES}

La producción promedio por lactancia fue de 6352,9 litros, con una desviación estándar de 3539,9 litros, lo que permitió un ingreso promedio de 2668,2 dólares con una desviación estándar de 1486,8 dólares, considerando como costos fijos: los servicios e impuestos y como costos variables: el mantenimiento de pastos y animales, el costo de producción promedio fue de 0,21 dólares con una desviación estándar de 0,10 dólares. Sin embargo, si se considera como costos fijos: mantenimiento de construcciones, mantenimiento de maquinaria y equipo, servicios e impuestos y como costos variables: establecimiento de pastos, mantenimiento de pastos y animales, así como la asistencia técnica, el costo de producción real promedio del litro de leche en la comunidad Sivicusig es de 0,43 dólares, con una desviación estándar de 0,19 dólares. Los pequeños productores al no ver la actividad pecuaria, como una empresa, no llevan los registros contables y por ello desconocen los costos reales de producción, razón por la cual, no identifican, si su actividad es rentable, no crecen y no llegan a ser competitivos.

\section{LISTA DE REFERENCIAS}

Britt, J., Thomas, R., Speer, N. and Hall, M. 2003, Efficiency of converting nutrient dry matter to milk in Holstein herds, J Dairy Sci, 86(11):3796-801

El Universo. 2019. ¿Bebida láctea o leche pura?, dilema del sector productivo en Ecuador.

[En línea]. Consultado el 11-11-2020. https://www,eluniverso,com/noticias/2019/09/07/nota/7506160/uso-suero-lechedivide-sector-lacteo

Federación Colombiana de Ganaderos - Fedegan. 2004. Cadenas productivas cárnica bovina y láctea de Estados Unidos. Oportunidades y Amenazas: Oficina de Planeación - Fedegan - F.N.G. Bogotá. D.C.; 52

GADMSIGCHOS. 2015. Actualización del plan de desarrollo y ordenamiento territorial del cantón Sigchos, [En línea]. Consultado el 09-11-2020. http://app,sni,gob,ec/sni-

link/sni/PORTAL_SNI/data_sigad_plus/sigadplusdiagnostico/0560001190001_c onsolidado\%20Diagn\%C3\%B3stico\%20Sigchos_15-03-2015_20-43-37,pdf 
Gómez, O., et. al. (2017). Análisis de rentabilidad de la producción de leche de acuerdo con la variación de la fuente de carbohidrato utilizada en el suplemento de vacas Holstein. Rev. Med. Vet. (34 Supl):9-22.

Hadley, G., Harsh, S., y Wolf, C. 2002. Managerial and financial implications of major dairy farm expansions in Michigan and Wisconsin. J. Dairy Sci;85(8):2053-64

El telégrafo. 2018. El litro de leche se paga a \$ 0,42 a los productores. [En línea]. Consultado el 16-11-2020. [internet]. www,eltelegrafo,com,ec

Ekos. 2019. Producción de leche en Ecuador. [En línea]. Consultado el 16-11-2020. https://www,ekosnegocios,com/articulo/produccion-de-leche-enecuador\#: :text=Una\%20industria\%20formal\%20debe\%20ajustarse,es\%20de\% 20USD $\% 200 \% 2 \mathrm{C} 42$,

El Mercurio. 2020. Panorama incierto para ganaderos. [En línea]. Consultado el 16-112020. https://ww2,elmercurio,com,ec/2020/07/15/panorama-incierto-paraganaderos/

Normas Internacionales de Información Financiera (NIIF). 2012. Propiedades, planta y equipo, NIC 16. International Financial Reporting Standards. [En línea]. Consultado el 16-11-2020. http://www,ifrs, org/IFRSs/IFRS-technicalsummaries/Documents/ Spanish2012/IAS16,pdf

Osorio, F. 2004. Efecto del manejo alimentario sobre el sistema especializado de producción lechera, En: Memorias Seminario Nacional de Lechería Especializada: Bases Nutricionales y su Impacto en la Productividad, Eventos y Asesorías Agropecuarias, 1-2; Medellín, Colombia.

Salazar, A. y Cochet, H. 2016. Haciendas y campesinos lecheros en el Carchi (Andes húmedos del norte del Ecuador): dinámica productiva y comparación de los resultados técnicos y económicos. Revista de Geografía Agrícola. Núm. 57. Pp. 7-25. Universidad Autónoma Chapingo Texcoco, México.

Torres, X. 2018. Estudio de la producción de la industria láctea del cantón Cayambe en el período 2009-2015. Tesis previa a obtener el título de magister en Administración de empresas. Universidad Andina Simón Bolívar.

Wolf, C. 2010. Understanding the milk to feed price ratio as a proxy for dairy farm profitability 